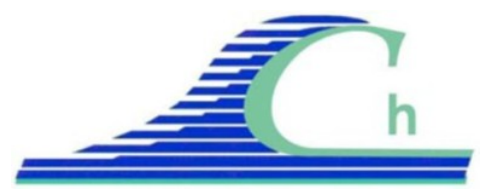

XII ${ }^{\text {ìmes }}$ Journées Nationales Génie Côtier - Génie Civil

Cherbourg, 12-14 juin 2012

DOI:10.5150/jngcgc.2012.056-V @ Editions Paralia CFL

disponible en ligne - http://www.paralia.fr - available online

\title{
Etude préalable à la prévention des submersions marines dans une zone sensible à l'érosion : la Belle Henriette (Vendée)
}

\author{
Cyril VANROYE ${ }^{1}$, Emmanuel DEVAUX $^{2}$, Patrick CHASSE $^{3}$
}

1. Direction Départementale des Territoires et de la Mer de la Vendée, Service Gestion Durable de la Mer et du Littoral, 1 quai Dingler, 85100 Les Sables d’Olonne, France. cyril.vanroye@vendee.gouv.fr

2. Centre d'Etudes Techniques de l'Equipement de l’Ouest, rue René Viviani, 44000 Nantes, France.

Emmanuel.devaux@developpement-durable.gouv.fr

3. Centre d'Etudes Maritimes et Fluviales, 2 boulevard Gambetta, 60200 Compiègne, France.

patrick.chasse@developpement-durable.gouv.fr

\section{Résumé :}

La Belle Henriette est un site lagunaire (une casse) situé au sud du département de la Vendée. Il s’étend sur une superficie d'environ 170 ha et se développe sur un linéaire de près de $4 \mathrm{~km}$ sur les communes de la Faute sur mer et de la Tranche sur mer. Le contexte morphologique de la Baie de l'Aiguillon et la formation de la Pointe d'Arçay (flèche sableuse dont l'allongement est de près de 20 mètres par an, sur la période 18241977) ainsi que les aménagements successifs, ont abouti à la création d'un espace lagunaire semi-fermé particulièrement riche sur la plan de la biodiversité. Cette richesse a été reconnue au travers du classement récent en Réserve Naturelle Nationale.

Lors des évènements tempétueux et notamment très récemment lors de la tempête Xynthia, la formation de brèches a permis l'intrusion d'un volume important d'eau de mer. Cet envahissement brutal a été à l'origine de la submersion des terrains situés au nord ouest de la commune de la Faute sur mer participant ainsi à la submersion généralisée du secteur. Suite à cet événement, une démarche de prévention des submersions marines a été initiée. Elle vise à développer le système de protection non plus au niveau du cordon dunaire de premier rang comme cela a été le cas par le passé mais en arrière de la lagune. Ce changement de stratégie tient autant aux retours d'expérience de la submersion Xynthia qu’à la volonté de préserver et de respecter le caractère lagunaire du site. La démarche passe ainsi par une description géomorphologique du site, une évaluation de l'aléa au travers de la modélisation des conséquences hydrauliques d'un événement de type Xynthia et des enjeux impactés. La mise en place d'un système de protection repose sur une approche intégrée visant à préserver les enjeux et à protéger les personnes mais aussi à sauvegarder le milieu naturel. 


\section{Mots-clés :}

Tempête marine - Vulnérabilité - Risques côtiers

\section{Introduction}

Le secteur d'étude se situe dans le département de la Vendée, respectivement à l'ouest et à l'est des communes de la Tranche sur Mer et la Faute sur Mer (figure 1). Il est constitué d'un milieu lagunaire fermé par une flèche sableuse qui s'est formée parallèlement au trait de côte sous l'effet de la dérive littorale. En partie submergé lors de la tempête Xynthia en février 2010, il constitue un milieu vulnérable car des enjeux socio-économiques importants y sont présents. L’étude vise à définir un programme d'actions de protection contre la submersion marine permettant d'assurer la protection des populations et de conserver les caractéristiques naturelles de l'espace lagunaire. Dans le présent article, on présentera successivement les caractéristiques du site et son fonctionnement géomorphologique, l'impact de la tempête Xynthia, la méthodologie d'analyse qui repose sur la modélisation hydraulique et l'analyse des enjeux. La conclusion de l'étude donnera les principes du programme d'action que l'analyse préalable aura permis de dégager.

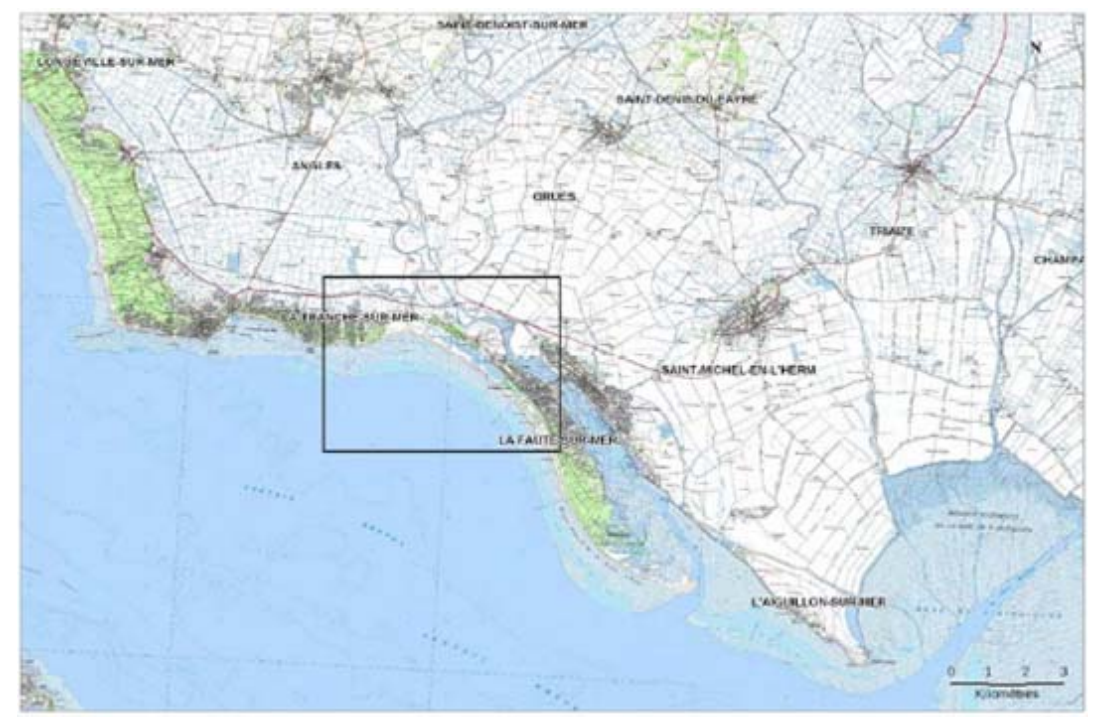

Figure 1. Localisation du site d'étude (@IGN scan 25®).

\section{Description du site}

La lagune de la Belle Henriette est le résultat d'une formation dunaire dans le pertuis breton. Cette zone humide s'étend sur $4 \mathrm{~km}$ de long et $500 \mathrm{~m}$ de large, soit une superficie d'environ 180 hectares. Elle est délimitée côté terre par une route départementale construite en grande partie sur l'ancien cordon littoral, surplombant le fleuve Le Lay qui s'écoule selon une direction nord-ouest/sud-est. Côté mer, le cordon dunaire plus récent qui sépare la lagune de l'océan est affecté par l’ouverture de brèches 


\section{XII ${ }^{\text {èmes }}$ Journées Nationales Génie Côtier - Génie Civil \\ Cherbourg, 12-14 juin 2012}

lors des plus fortes tempêtes. Globalement, la dynamique sédimentaire de ce littoral se caractérise par une importante migration du sable vers le sud-est (DHI \& GEOS, 2007). L'érosion se traduit par un recul du trait de côte plus marqué dans la partie ouest du secteur. A l'extrémité sud, l'accumulation des sédiments entraîne l'allongement permanent et rapide de la flèche sableuse de la Pointe d'Arçay (plus de $5 \mathrm{~km}$ depuis 1750 ; WEBER, 2004). Cette lagune est complètement séparée de l’océan par le cordon dunaire depuis 1971. Elle bénéficie d'apports d'eau salée lors des tempêtes, par surverse, ce qui a permis la mise en place d'un habitat spécifique et lui confère un intérêt particulier. Cette richesse écologique a prévalu à sa désignation en tant que site NATURA 2000 et plus récemment comme réserve naturelle nationale. Ce site est au coeur d'enjeux de conservation d'habitats et de menaces liées à l'intervention humaine (fréquentation inorganisée du site, travaux de défense contre la mer, etc.).

\section{Evolution géomorphologique du site}

Le site de la Belle Henriette a connu de profondes modifications (figure 2) depuis la fin du 19ème siècle, sous l'action des forçages naturels et anthropiques (DEAT, 1995; MUSEREAU, 2009).

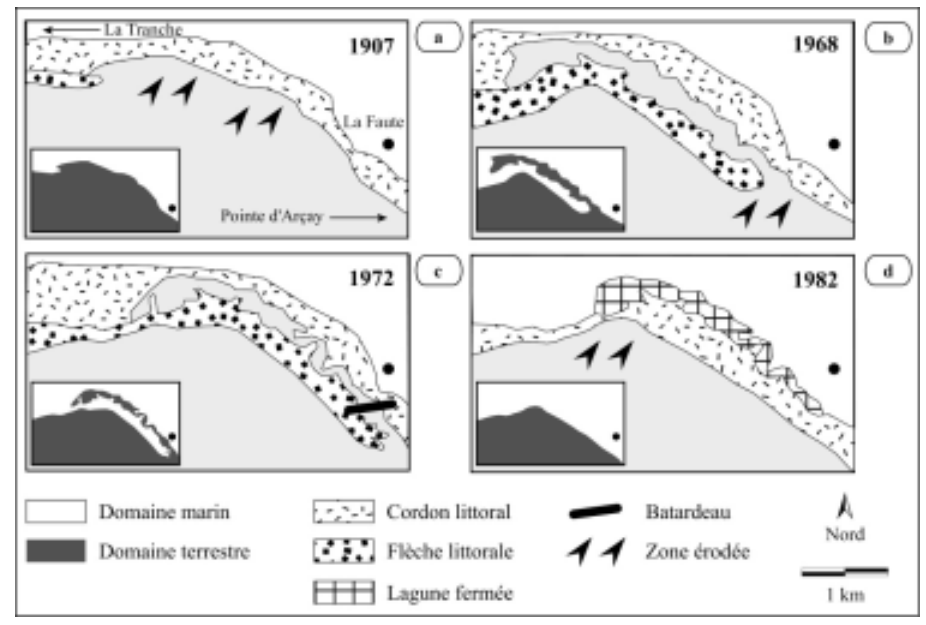

Figure 2. Formation de la Belle Henriette (DEAT, 1995 ; MUSEREAU, 2009).

Vers la fin du 19ème siècle, le secteur de la Belle Henriette est le siège d'une érosion très rapide. La croissance de la flèche sableuse sous l'effet d'une dérive littorale intense à partir de la Tranche sur mer a pour effet de déplacer le fulcrum vers le sud-est. Les tempêtes ouvrent des brèches régulièrement et des opérations de fermeture sont systématiquement entreprises. La flèche sableuse continuant de progresser, la lagune de la Belle Henriette est isolée des fortes agitations et est de moins en moins alimentée en eau de mer ; elle devient le siège d'une sédimentation sablo-vaseuse. En 1971, une digue de sable appelée "batardeau" est alors édifiée pour obstruer et ainsi lutter contre l'érosion qui touche l'est de l'exutoire. Après cette stabilisation du trait de côte, le 
phénomène d'érosion dû au transit littoral reprend, avec une grande sensibilité du littoral à l'extrémité nord-ouest (Rouillères).

\section{La tempête Xynthia}

Dans la nuit du 27 au 28 février 2010, la tempête Xynthia a affecté de manière importante les côtes du département de la Vendée (CHAUVEAU et al., 2011). Plus particulièrement dans le secteur d'étude où elle a provoqué l'ouverture de brèches dans le cordon dunaire et des submersions dans le secteur de la Belle Henriette (figure 3).

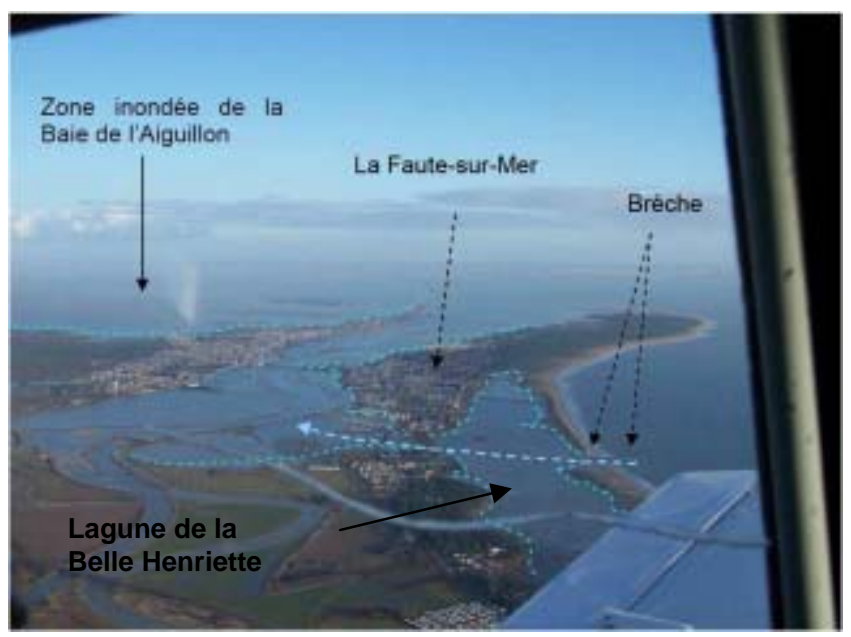

Figure 3. Intrusion d'eau de mer lors de Xynthia (BRGM, 2010).

Suite à la tempête, 14 relevés de laisses ont été effectués le long des ouvrages de protection et de certains chemins d'accès aux plages. Les altitudes relevées aux extrémités est et ouest de la lagune sont d'environ 4,20 m NGF, et dépassent 5 m NGF dans la zone centrale proche du quartier des Vieilles Maisons. Treize brèches ont été constatées (figure 4). Sept brèches se sont formées (brèches 1 à 7) au droit du quartier des Rouillères à la Tranche sur Mer, submergé lors de la tempête Xynthia. Les brèches $9,10,11$ et 13 se sont ouvertes au niveau de chemins d'accès à la plage. La brèche $12 \mathrm{a}$ été ouverte dans un secteur où le cordon ne semblait pas présenter de faiblesse particulière. Il est à noter que la brèche 13 , au niveau du Platin, a formé un chenal de communication permanent entre la mer et la lagune et que des travaux d'urgence (mise en oeuvre de bigbag remplis de sable) ont été réalisés afin de stopper le phénomène. Ces brèches ont permis l'intrusion d'une quantité importante d'eau submergeant un lotissement, un camping et la RD 46. 


\section{XII ${ }^{\text {èmes }}$ Journées Nationales Génie Côtier - Génie Civil \\ Cherbourg, 12-14 juin 2012}

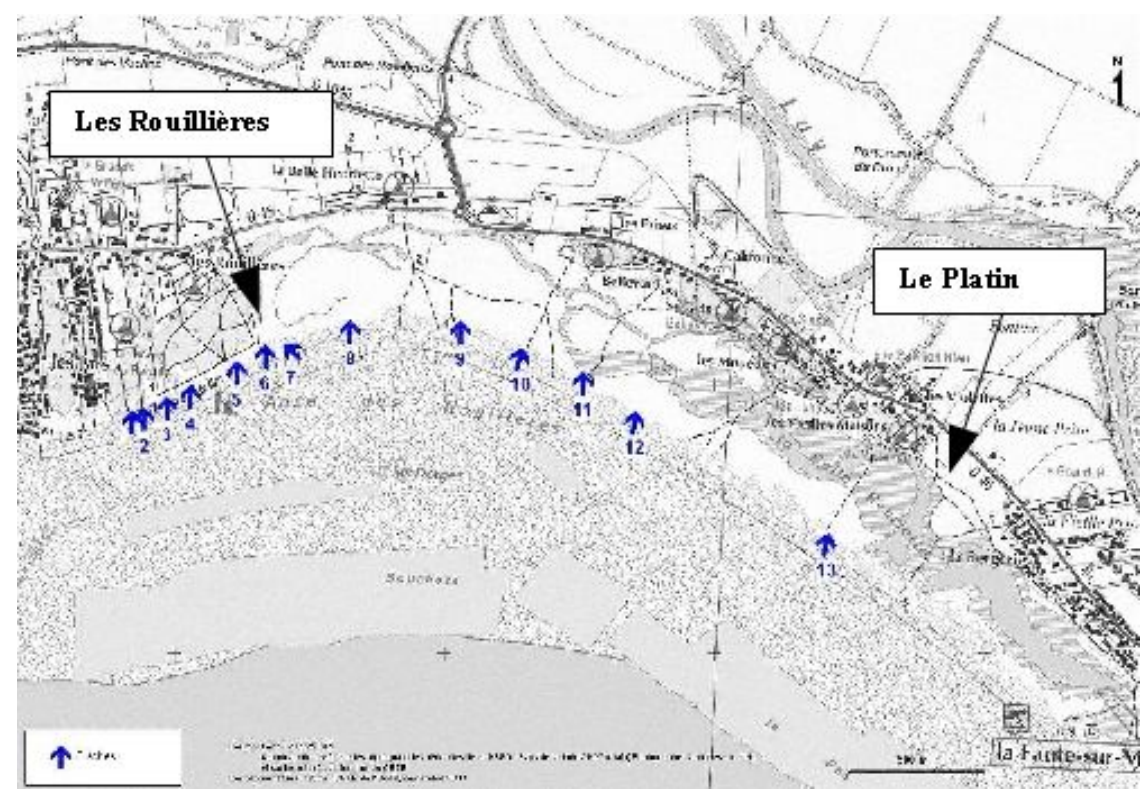

Figure 4. Localisation des brèches lors de Xynthia.

Les relevés altimétriques de ces laisses de submersion ont permis de cartographier les hauteurs d'eau. Le zonage établi met en avant que les hauteurs d'eau inférieures à 0,50 m représentent une grande partie de la zone d'étude. Les hauteurs d'eau comprises entre $0,50 \mathrm{~m}$ et $1 \mathrm{~m}$ ont été atteintes au coeur de la zone et dans une petite partie au milieu de l'espace précédent. Les terrains soumis à des hauteurs d'eau supérieures à $1 \mathrm{~m}$ sont situés le long de la rive du Lay. Il est cependant difficile d'identifier la contribution respective des eaux en provenance de l'estuaire du Lay et de celles en provenance de la lagune de la Belle Henriette.

\section{Modélisation des épisodes de submersion}

Afin d'apprécier l'impact des submersions, une modélisation hydraulique a été réalisée à l'aide du code de calcul Telemac2D développé par EDF-LNHE et un consortium de partenaires français et européens. Le modèle numérique de terrain est issu du relevé Lidar effectué à l'été 2010 réalisé par l’IGN dans le cadre de Litto3D®. L'effet d'un épisode tempétueux de référence avec un niveau d'eau maximal de 4,70 m NGF atteint au cours d'un cycle de marée a été modélisé par le CETMEF. Cela consiste à forcer le modèle hydraulique avec un hydrogramme de marée (figure 5). 


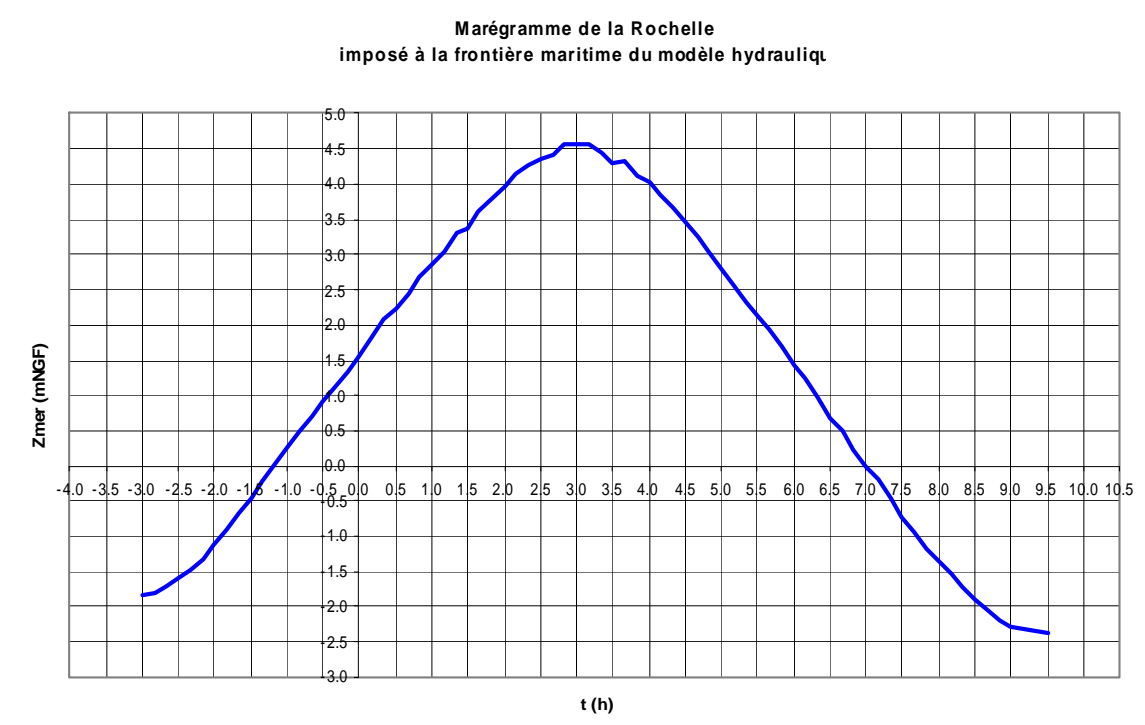

Figure 5. Marégramme imposé à la frontière du modèle hydraulique.

Cette méthode présente l'intérêt d'être relativement simple en termes de mise en œuvre par rapport à des modélisations plus complexes (propagation de la houle, prise en compte des effets du vent et du déferlement, etc.). Compte tenu de l'évolution morphologique du site et des retours d'expérience des dernières tempêtes, le cordon littoral de $1^{\text {ère }}$ ligne ne peut constituer une protection efficace. Dans le cadre de cette étude, l'objectif de la modélisation était d'évaluer la fiabilité du système en déterminant l'impact d'une défaillance. Trois scénarios relatifs à la fiabilité du système, décrits cidessous, ont donc été envisagés :

- Scénario 1 : Le site est considéré être tel qu'il se trouvait en été 2010 : brèches de la tempête Xynthia refermées et cordon dunaire partiellement renforcé ; on suppose que le cordon dunaire et les ouvrages jouant un rôle de protection ne présentent aucune faiblesse.

- Scénario 2: L'hypothèse est faite que le cordon dunaire de premier rang est inexistant ; l'Anse des Rouillères est dans ce cas ouverte jusqu'à la grande plage de La Faute. La morphologie a donc été modifiée afin d'araser ce cordon. L'altitude du terrain sur l'emprise de cordon est fixée à 1,5 m NGF

- Scénario 3 : Le scénario 2 est repris en considérant que des brèches se produisent dans les ouvrages de protection à deux endroits sur un linéaire côtier de $100 \mathrm{~m}$. L'une au niveau de la dune de la Bergerie, l'autre à l'extrémité ouest de l'ouvrage longeant la RD46. Elles seront supposées se produire 1h avant le niveau de pleine mer, soit à un niveau d'environ $4 \mathrm{~m}$ NGF. La cote d'arase au niveau de la brèche est fixée à $2 \mathrm{~m}$ NGF. 


\section{XII ${ }^{\text {èmes }}$ Journées Nationales Génie Côtier - Génie Civil \\ Cherbourg, 12-14 juin 2012}

La localisation des brèches repose sur l'analyse historique ainsi que sur le diagnostic détaillé du système de protection actuel réalisé dans le cadre de l'étude (CETE DE L'OUEST, 2010a ; 2010b).

\section{Eléments d'analyse de la submersion marine et de la vulnérabilité des enjeux}

Le cordon dunaire de premier rang, même s'il ne peut empêcher l'envahissement de la lagune par les eaux marines, a un rôle important contre la submersion.

Dans le scénario 1 (figure 6), il permet en effet de contenir la submersion dans la zone lagunaire, et évite le franchissement du cordon "de seconde ligne" situé en arrière de cette dernière.

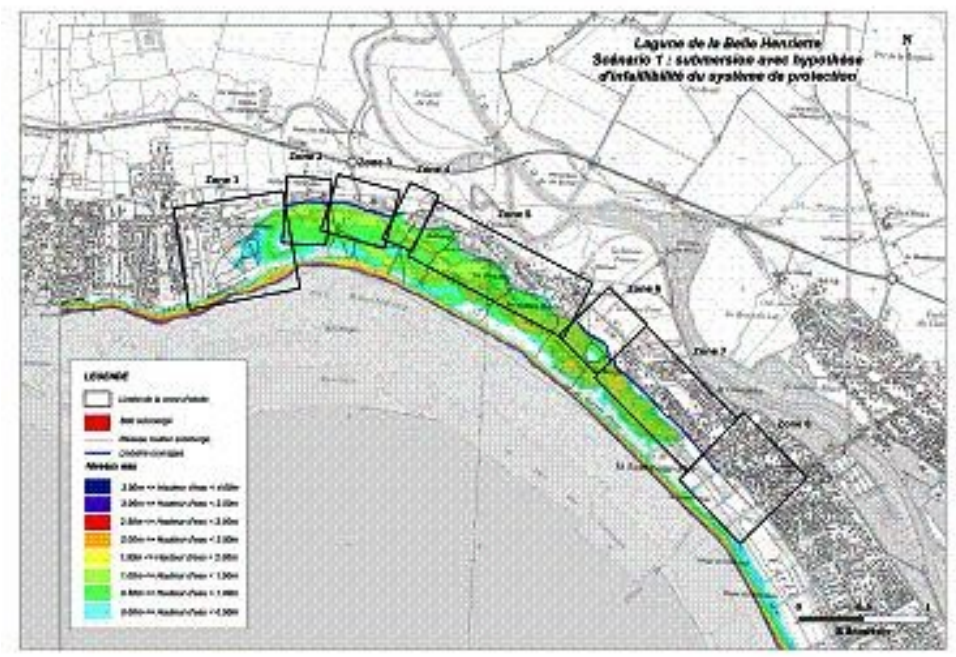

Figure 6. Résultats de la modélisation hydraulique - Scénario 1.

Très peu de bâtiments parmi ceux situés immédiatement en périphérie de la lagune sont submergés. Quelques bâtiments sont toutefois touchés au niveau de la Tranche sur mer. Il est important de noter que la RD 46, voie d'accès principale à la zone, n'est quasiment pas franchie. Les scénarios 2 et 3 font apparaître une submersion bien plus étendue et d'autant plus importante lorsque les brèches sont présentes au niveau du cordon littoral situé en arrière de la lagune (cf. scénario 3). 


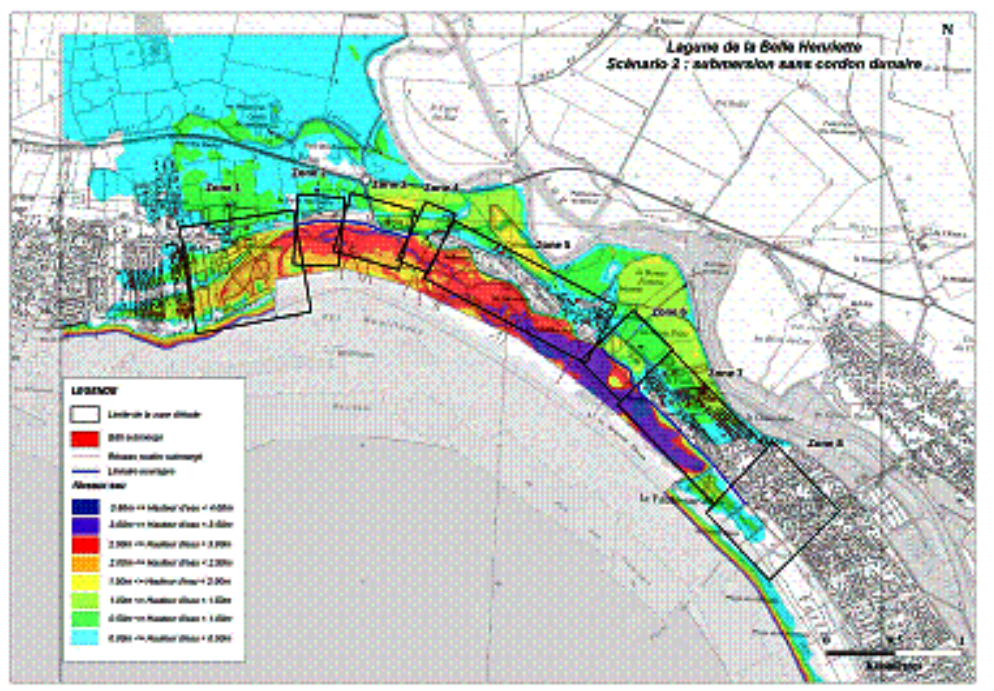

Figure 7. Résultats de la modélisation hydraulique - Scénario 2.

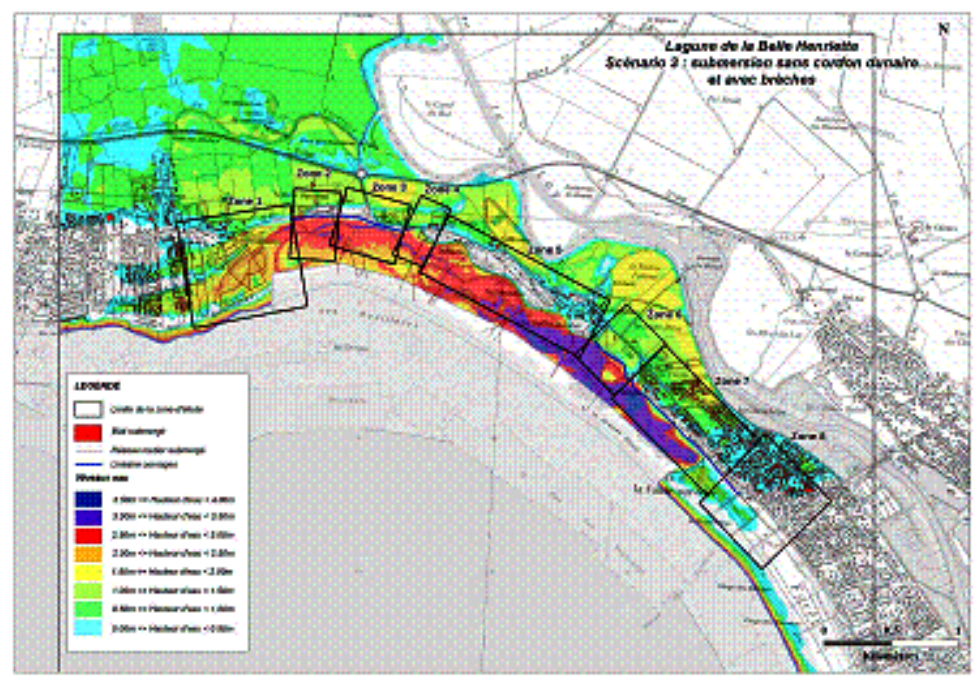

Figure 8. Résultats de la modélisation hydraulique - Scénario 3.

Les principaux secteurs d'habitations sont dans ce cas touchés par la submersion. Il s'agit principalement de la zone ouest des Rouillères (commune de la Tranche sur Mer), des Violettes plus à l'Est et du Nord du Bourg de la Faute sur Mer. Les zones touchées ne rejoignent toutefois jamais les zones "noires" où l'Etat a réalisé des acquisitions amiables et engagé des expropriations afin de mettre en les populations. La RD 46 est submergée sur plusieurs tronçons. La présence de brèches multiplie par 1,8 le nombre de bâtiments inondés qui atteint 1000 unités et fait passer le linéaire de voierie submergée d'environ 1,5 km à $2 \mathrm{~km}$. La hauteur d'eau dans les zones habitées dépasse $50 \mathrm{~cm}$ voire $1 \mathrm{~m}$ ponctuellement. On constate aussi l'effet "cuvette" associé aux digues du fleuve Lay au nord de la zone d'étude qui n’ont pas fait l'objet de rupture simulée. 


\section{XII ${ }^{\text {èmes }}$ Journées Nationales Génie Côtier - Génie Civil \\ Cherbourg, 12-14 juin 2012}

En dehors de la zone d'étude, aucune hypothèse de brèche n'a été faite sur les ouvrages de protection existant. La concomitance d'une crue du Lay et d'une submersion marine n’est pas envisagée dans cette approche.

\section{Définition d'un programme de protection}

Sur la base de ces éléments d'analyse, un programme de protection va pouvoir être bâti. Deux scénarios relatifs à la dynamique morphosédimentaire du cordon littoral seront envisagés :

- Maintien du cordon littoral de premier rang et hypothèses de brèches : cela suppose qu'il sera entretenu (mise en place de végétation, pose de ganivelles et rechargement régulier du cordon et en cas de détérioration après une tempête) de manière à maintenir une morphologie proche de l'état actuel. Cette hypothèse nécessite de pouvoir maintenir dans la durée un cordon dunaire par nature mobile ce qui est par définition impossible avec des techniques de gestion du trait de côte dites douces.

- Disparition du cordon dunaire : l'action conjuguée du vent, des vagues et du transit sédimentaire conduise à la disparition progressive du cordon dunaire, le site se retrouvant dans une configuration similaire à celle de la fin du $19^{\text {ème }}$ siècle. La défense du littoral repose alors sur la protection du cordon littoral "de seconde ligne" situé en arrière de la lagune. Il sera reprofilé en conséquence (en particulier pour résister aux chocs liés aux vagues et à une circulation d'eau régulière).

L'évaluation des deux scénarios sera réalisée au moyen d'une analyse multi-critères qui prendra en compte les éléments financiers proportionnellement au coût des enjeux menacés ; elle devra aussi prendre en compte l'intérêt environnemental et sociétal de la lagune (biodiversité associée au caractère lagunaire, usages chasse et baignade, etc.). Ces éléments sont discutés avec les acteurs locaux dans une approche de gouvernance participative comme le recommande les PAPI (programmes d'action de prévention des inondations) au niveau des territoires soumis au risque d'inondation.

\section{Références bibliographiques}

BRGM (2010). Tempête Xynthia: compte rendu de mission préliminaire. Mars, 53 p CETE DE L'OUEST -Laboratoire régional d'Angers- (2010a). Diagnostic des ouvrages de protection suite à l'évènement Xynthia. Mars 2010, Fiches de visites.

CETE DE L'OUEST -Laboratoire régional d'Angers- (2010b). Visites de diagnostic suite aux travaux de confortement. Octobre 2010, Fiches de visites.

DEAT E. (1995). Morphogenèse et évolution récente de la flèche sableuse et de la lagune de la Belle Henriette (Vendée): Proposition de restauration et de gestion du site. Mémoire de DEA, Université de Bretagne Occidentale, 142 p.

DHI, GEOS (2007), Étude de connaissance des phénomènes d'érosion sur le littoral vendéen, DDE de la Vendée, 348 p. 
Thème 2 - Dynamique sédimentaire

CHAUVEAU E., CHADENAS C., COMENTALE B., POTTIER P., BLANLEIL A., FEUILLET T., MERCIER D., POURINET L., ROLLO N., TILLIER I., TROUILLET B. (2011). Xynthia : leçons d'une catastrophe. Cybergeo : European Journal of Geography, article 538, doi:10.4000/cybergeo.23763

MUSEREAU J. (2009). Approche de la gestion des cordons littoraux: Mise au point et application d'un indice d'érosion (Zone des Pertuis Charentais, France), Thèse, Université Européenne de Bretagne Rennes 2, 306 p.

WEBER N. (2004). Morphologie, architecture des dépôts, évolution séculaire et millénaire du littoral Charentais : Apports de la sismique réflexion combinée à des suivis bathymétriques et validée par des vibrocarottages, Thèse, Université de La Rochelle, 372 p. 